\title{
Ensinando a Pesquisar: os Desafios diante da Diversidade da Psicologia
}

\author{
Teaching to Research: Challenges before the Diversity of Psychology \\ Enseñando a Investigar: los Desafios frente a la Diversidad de la Psicología
}

\author{
Cecilia Pescatore Alves' \\ Sandra Macedo Bettoi" \\ Mônica Helena Tieppo Alves Gianfaldoni"l' \\ Maria da Graça Marchina Gonçalves ${ }^{\text {IV }}$
}

Curso de Psicologia - Faculdade de Ciências Humanas e da Saúde Pontifícia Universidade Católica de São Paulo

E-mail: 'cpescatore@uol.com.br, "sanbettoi@uol.com.br, "'mhtag@uol.com.br, "vgrajota@uol.com.br

\section{Resumo}

O artigo trata do ensino de pesquisa, no âmbito de uma disciplina obrigatória de um curso de Psicologia, procurando, além de compartilhar um modo de fazer, evidenciar a perspectiva de formação em Psicologia presente na proposta. A disciplina parte do pressuposto de que o exercício de realizar uma pesquisa completa permite vivenciar a produção de conhecimento como processo de tomada de decisões, o que permite debater tanto pesquisas quanto o papel do pesquisador. A discussão teórica, que ocorre paralelamente aos exercícios, permite abertura para a diversidade de pesquisas em Psicologia em função de distintos pressupostos epistemológicos e metodológicos. Ao final de dois semestres, os estudantes de terceiro e quarto períodos deverão ser capazes de: (1) analisar diversas maneiras de pesquisar em Psicologia, tendo como referencial várias abordagens metodológicas presentes nas diferentes teorias da Psicologia e (2) realizar uma pesquisa, participando de todas as etapas desse processo.

Palavras-chave: construção do conhecimento, pesquisa científica, formação do psicólogo, estudantes universitários.

Abstract

This paper approaches the research teaching, as an obligatory subject of a Psychology degree. It aims, beyond the sharing of a how-to for the matter, to reveal the perspective of Psychology education that relies in the proposition. The discipline assumes that the exercise of completing a research allows the achiever to experience the production of knowledge as a process of decision taking, which makes possible to debate on the researches and on the researcher's role as well. The theoretical discussion, occurring concomitantly to the exercises, allows expanding the research diversity in Psychology due to distinct epistemological and methodologic premises. At the end of two semesters, the students of third and fourth terms must be able to: (1) analyze diverse manners to research in Psychology, having as references, various methodologic approaches contained in the different Psychology theories, and (2) fulfill a research, engaging to all steps of the process.

Keywords: construction of knowledge, scientific research, psychologist's education, college students.

\section{Resumen}

El artículo trata de la enseñanza de la investigación, en el ámbito de una disciplina obligatoria de una carrera de Psicología, buscando, además de compartir un modo de hacer, evidenciar la perspectiva de formación en Psicología presente en la propuesta. La disciplina presupone que el ejercicio de realizar una investigación completa permite vivenciar la producción de conocimiento como proceso de toma de decisiones, lo que permite debatir tanto las investigaciones como el papel del investigador. La discusión teórica, que sucede paralelamente a los ejercicios, permite dar a conocer la diversidad de investigaciones en Psicología en función de distintos presupuestos epistemológicos y metodológicos. Al final de dos semestres, los estudiantes del tercero y cuarto semestre deberán ser capaces de: (1) 
analizar diversas maneras de investigar en Psicología, tomando como referencia varios abordajes metodológicos presentes en las diferentes teorías de la Psicología y (2) realizar una investigación, participando de todas las etapas de ese proceso.

Palabras-clave: construcción del conocimiento, investigación científica, formación del psicólogo, estudiantes universitarios.

A formação em Psicologia tem muitos desafios. Parte deles refere-se às características da área, marcada pela diversidade epistemológica, teórica, de campos de atuação e tipos de práticas. Outra parte diz respeito ao contexto em que se situa a formação em nível superior no Brasil, atravessado por interesses também diversos e pautado por concepções que situam a educação no campo dos direitos sociais ou no campo dos negócios, o que traz implicações para as condições de implementação dessa formação.

São desafios, também, as demandas decorrentes da realidade brasileira, quando se pretende pautar a formação de profissionais pelo compromisso com questões sociais relevantes e com ações que coloquem a ciência e a atividade profissional a serviço do enfrentamento dessas questões. Um cenário assim complexo requer reflexões que extrapolem procedimentos pedagógicos e técnicas de ensino, embora esses elementos sejam fundamentais para garantir qualquer formação de qualidade.

A Psicologia tem um histórico de debates sobre a nossa ciência e profissão que começa pela afirmação de princípios que possam fazer frente à complexidade da formação. A Lei 4.119, de 27 de agosto de 1962, regulamentou a profissão do psicólogo, e o currículo mínimo para os cursos de Psicologia foi fixado em dezembro de 1962 por Resolução do Conselho Federal de Educação. Como consequência da Constituição de 1988 e de novas perspectivas para a Educação, a formação estava de novo em pauta. Em um encontro nacional ocorrido em 1992, em São Paulo, congregando o Conselho Federal e Conselhos Regionais de Psicologia, além de 98 - de 103 - Instituições de Ensino Superior, foi produzida e publicada a Carta de Serra Negra, com "Diretrizes Gerais para a Formação em Psicologia" (Bernardes, 2004; Conselho Regional de Psicologia de São Paulo, 1998/2016). Outros debates importantes ocorridos no período subsequente produziram documentos firmados em princípios de formação, especialmente considerados a partir da Lei de Diretrizes e Bases para a Educação Nacional (1996). O encontro de profissionais, pesquisadores e docentes chamado Fórum Nacional de Formação ${ }^{1}$ (ocorrido em 1997, em Ribeirão Preto) produziu o documento "Princípios para a Formação em Psicologia", que foi referência para os debates durante o processo de elaboração das Diretrizes Curriculares Nacionais (DCN) ${ }^{2}$ (Conselho Regional de Psicologia, 1998).

Os princípios explicitados nesse Fórum de Formação foram os seguintes: formação básica, pluralista e sólida; formação generalista; formação interdisciplinar; formação que prepare para a atuação profissional; formação científica, crítica e reflexiva; integração teoria e prática; compromisso com o atendimento das demandas sociais; compromisso ético; ruptura com o modelo tecnicista; construção de uma identidade profissional.

Por sua vez, também as DCN apresentam princípios para a formação e é interessante atentar para o fato de que esse item, explicitação

10 Fórum Nacional de Formação foi promovido pelo Conselho Federal de Psicologia e com outras entidades que naquele momento formavam o Fórum de Entidades Nacionais da Psicologia Brasileira (FENPB).

2 As Diretrizes Curriculares Nacionais da Psicologia (DCN) tiveram uma primeira versão aprovada em 2004, após um longo processo de elaboração e discussão que envolveu as entidades de Psicologia. As DCN atuais estão na Resolução CNE/CES n. 05/2011. 
de princípios, não é obrigatório nem habitual em diretrizes de outras áreas. Esses princípios estão no artigo $3^{\circ}$ da Resolução CNE/CES n. 08/2004 (primeira versão das DCN), mantido como artigo $3^{\circ}$ da Resolução em vigor (Brasil, 2011). São eles:

I - construção e desenvolvimento do conhecimento científico em Psicologia; II compreensão dos múltiplos referenciais que buscam apreender a amplitude do fenômeno psicológico em suas interfaces com os fenômenos biológicos e sociais; III - reconhecimento da diversidade de perspectivas necessárias para compreensão do ser humano e incentivo à interlocução com campos de conhecimento que permitam a apreensão da complexidade e multideterminação do fenômeno psicológico; IV - compreensão crítica dos fenômenos sociais, econômicos, culturais e políticos do país, fundamentais ao exercício da cidadania e da profissão; $V$ - atuação em diferentes contextos, considerando as necessidades sociais e os direitos humanos, tendo em vista a promoção da qualidade de vida dos indivíduos, grupos, organizações e comunidades; VI - respeito à ética nas relações com clientes e usuários, com colegas, com o público e na produção e divulgação de pesquisas, trabalhos e informações da área da Psicologia; VII - aprimoramento e capacitação contínuos.

\section{Ensinar a fazer e fazer ensinando}

A experiência aqui relatada refere-se ao Curso de Psicologia da PUC/SP, mais especificamente às disciplinas Modelos de Investigação I e II, de caráter obrigatório, oferecidas no $3^{\circ}$ e $4^{\circ}$ períodos da graduação em Psicologia. As duas disciplinas são sequenciais, ou seja, Modelos de Investigação I é pré-requisito para Modelos de Investigação II; elas têm natureza teórico-prática; ocorrem em turmas de 20 alunos; e têm duração de 57 horas cada uma. Essas duas disciplinas são de responsabilidade de uma equipe de professores,
$\mathrm{O}$ que queremos indicar com as referências a esses processos de discussão são dois aspectos: a presença mesma do debate, da preocupação com a formação no âmbito das entidades da psicologia; e a explicitação de princípios, no sentido de garantir uma proposta educacional ampla e não uma perspectiva tecnicista de formação. Vale lembrar que os dois apontamentos têm relação com a Associação Brasileira de Ensino de Psicologia (ABEP), pois a criação da entidade foi uma resposta à preocupação com esse tema; e os espaços da entidade têm permitido cuidar da formação superando visões pragmáticas e imediatistas (um exemplo disso é a Carta de Salvador, produzida com o Fórum de Coordenadores de Curso de Psicologia, em 2011, resgatada no sítio da Associação Brasileira de Ensino de Psicologia, 2016).

Essas considerações iniciais pretendem situar a experiência que aqui será relatada, também como referida em princípios, no contexto mais geral da área e seus desafios para a formação. Trata-se do ensino de pesquisa, no âmbito de uma disciplina obrigatória de um curso de Psicologia, procurando, além de compartilhar um modo de fazer, evidenciar a perspectiva de formação em Psicologia presente na proposta.

com diferentes afiliações teóricas e experiência em pesquisa ${ }^{3}$.

O desenho geral da disciplina inclui a apresentação de diferentes concepções de método científico e seus desdobramentos para a condução do processo de pesquisa; além da experiência prática de realização de uma pesquisa. Ao final dos dois semestres, os estudantes deverão:

Analisar diversas maneiras de pesquisar em Psicologia, tendo como referencial várias abordagens metodológicas presentes nas

3 Compõe a Equipe de Professores responsáveis pela disciplina, além das autoras do texto, os professores: Denize Rosana Rubano, Fabíola Freire Saraiva de Melo, Marcelo Sodelli, Maria de Lourdes Bara Zanotto, Paulo José de Carvalho e Sandra Gagliardi Sanchez. 
diferentes teorias da Psicologia.

Realizar uma pesquisa, participando de todas as etapas desse processo.

Para alcançar os objetivos, e considerando sua natureza teórico-prática, a disciplina aborda tanto conteúdos teóricos quanto conteúdos práticos. Considera-se parte dos conteúdos teóricos:

A pesquisa como processo não linear de tomada de decisão;

Características gerais das diversas etapas da pesquisa;

Pressupostos epistemológicos e características de diferentes modelos de pesquisa em Psicologia: Modelo de pesquisa em Ciências Naturais, Modelo de pesquisa em Fenomenologia, Modelo de pesquisa em Psicanálise, Modelo de pesquisa em Análise do Comportamento e Modelo de pesquisa em Psicologia Sócio-histórica.

Para trabalhar como realizar uma pesquisa em todas as suas etapas, os conteúdos são:

- A escolha do tema de pesquisa;

- Revisão bibliográfica;

- As normas de elaboração de referências bibliográficas;

- A elaboração de resumos e citações;

- A colocação do problema de pesquisa e a justificativa de sua relevância científica e social;

- O planejamento da pesquisa: previsão de análise e plano de coleta;

- Coleta de informações;

- Análise de dados: categorização, classificação, agrupamento, representação e síntese das informações coletadas; descrição dos resultados e estabelecimento de relações;

- Interpretação dos dados;

- Comunicação da pesquisa: elaboração de relatório e apresentação.

As estratégias de aula são diversas, de modo a possibilitar o contato do aluno com diferentes maneiras de pesquisar em Psicologia, conhecer e debater os fundamentos epistemológicos que as sustentam e fazer um exercício de pesquisa, percorrendo todas as suas etapas. Predominam as seguintes atividades: leitura e discussão de relatos de pesquisa de diferentes abordagens do fenômeno psicológico; leitura e discussão de material teórico sobre as diferentes maneiras de pesquisar em Psicologia; atividades práticas relativas a etapas do processo de pesquisa.

A disciplina considera e procura concretizar os princípios educacionais que estão no projeto pedagógico do curso. São eles: garantir uma formação teórica sólida e a articulação teoria e prática; possibilitar a formação em pesquisa; propiciar reflexão sobre o papel do pesquisador; propiciar reflexão sobre a intervenção em Psicologia; estabelecer relação com a realidade brasileira; garantir a participação do aluno.

As características da disciplina descritas acima podem ser avaliadas em função desses princípios. Assim, a fim de garantir uma formação teórica sólida e a articulação teoria e prática, podemos ver que o curso enreda o fazer pesquisa com a discussão teórica dos fundamentos de diferentes modelos de investigação. Os objetivos articulam o aprendizado de conceitos teóricos com sua aplicação na realização da pesquisa; e as estratégias envolvem aulas e textos teóricos, aulas e textos sobre o processo de pesquisa, exercícios relativos às diferentes etapas da pesquisa e realização de uma pesquisa em grupo.

Não se trata de uma articulação simples, pois há vários elementos que tornam complexa sua realização. Um deles é a natureza da discussão teórica, que, ao mesmo tempo em que envolve alto nível de abstração, só tem sentido quando compreendida nas implicações concretas para a produção de conhecimento. Os recursos utilizados em relação a isso contam com disciplinas anteriores, que são pré-requisitos para esta: Bases Epistemológicas e História da Psicologia. Além disso, recorre-se à leitura de muitos relatos de pesquisa, que explicitem o fazer de cada modelo de investigação, procedendo-se a uma leitura reflexiva e crítica que permita ao aluno reconhecer tanto as decisões, critérios e procedimentos relatados, quanto à adesão do pesquisador aos pressupostos e características de um determinado modelo. 
Outra dificuldade decorre do fato de que o aluno deve entrar em contato com os conceitos teóricos pertinentes a cada abordagem, relativamente à sua compreensão do que é o pesquisar e, ao mesmo tempo, paralelamente, realizar uma pesquisa, desde a escolha do tema, passando pela revisão de literatura, colocação do problema, planejamento, coleta de informações, análise e interpretação de dados e comunicação. São processos de aprendizagem que ocorrem simultaneamente, o teórico e o prático, de forma que o aluno não opta por um modelo para realizar sua pesquisa. O que faz, então? Isso não seria incoerente com a perspectiva de apresentar diversas maneiras de pesquisar em Psicologia? Não seria incoerente, também, com o princípio de garantir articulação teoria e prática?

A solução encontrada no desenvolvimento da disciplina tem sido trabalhar no exercício prático de realização de uma pesquisa aquilo que denominamos de fundamentos práticos do pesquisar, ou seja, atividades que levem ao desenvolvimento de habilidades básicas, tais como: levantar aspectos que permitam identificar temas de pesquisa em Psicologia; estabelecer relações entre esses temas e a produção teórica e de pesquisa em Psicologia; identificar fatores que atestem a relevância do tema; realizar atividades de revisão de literatura, em diversas modalidades e amplitudes; formular questões que levem ao problema de pesquisa; identificar informações que podem responder ao problema de pesquisa e planejar a maneira de obtê-las; produzir resultados baseados nas informações obtidas e organizadas com critérios claros e de maneira sistemática; ampliar o significado desses resultados com informações obtidas em outras pesquisas ou junto a produções teóricas pertinentes; comunicar de forma clara todo o processo de pesquisa. E, principalmente, saber definir e defender critérios para a tomada de decisão em cada uma dessas atividades básicas.

Na medida em que o curso avança e os modelos são discutidos teoricamente, essas atividades podem incluir reflexões a partir dos diferentes modelos de investigação, à guisa de comparação entre possibilidades existentes, sem ainda significar ou exigir escolha de um deles.

Dessa forma, a apresentação da diversidade de modelos é um constante desafio. Um dos subsídios nessa discussão, como dito acima, é relacionar as características de cada modelo com suas bases epistemológicas. Ao mesmo tempo, a proposta é apresentar a diversidade e fazer a pesquisa sem "escolha" de um modelo, por meio do ensino dos fundamentos práticos do pesquisar.

Deve-se também apresentar as diferenças entre os modelos sem definir preferências ou avaliações. A legitimidade de todos deve ser fundamentada no desenvolvimento histórico do conhecimento e os critérios de comparação devem ter base nos fundamentos históricos e epistemológicos. Para garantir isso, todos os modelos são apresentados por meio dos mesmos itens, a saber: contextualização histórica e no processo de discussão epistemológica e teórica; a finalidade da ciência para determinado modelo; concepção de relação sujeito-objeto; concepção de objeto da Psicologia; concepção sobre o papel da teoria e da empiria; concepção de generalização do conhecimento produzido; principais procedimentos de pesquisa.

Em relação aos princípios do currículo possibilitar a formação em pesquisa; propiciar reflexão sobre o papel do pesquisador; propiciar reflexão sobre a intervenção em Psicologia; estabelecer relação com a realidade brasileira - entendemos que eles são garantidos na medida em que o objeto da disciplina é o processo de pesquisa, no âmbito teórico e prático. Além disso, como parte integrante do processo de pesquisa, a relevância social do problema é sempre enfatizada; a título de exemplo dessa preocupação, um dos exercícios que os alunos realizam é a busca em sítios de entidades da Psicologia para identificar a quais debates a categoria dos psicólogos tem se dedicado.

Todos os estudantes terminam as disciplinas apresentando pôsteres dos seus trabalhos de pesquisa em evento aberto a toda comunidade da universidade, com apresentação oral e debatedores convidados. É o momento de cumprir a última etapa do processo de construção do conhecimento 
científico em que a comunicação dos resultados e a exposição à crítica construtiva de outros pesquisadores evidenciam que a ciência é feita coletivamente.

\section{Tecendo considerações finais}

Uma vez que a universidade tem como função a formação e a produção do saber, entendemos que espaços de valorização da pesquisa contribuem para a visão crítica do aluno, pois se trabalha com diversas noções que contribuem para isso: o conhecimento científico e a própria noção de ciência se alteram, têm caráter histórico. Por isso, a pesquisa constante é fundamental.

$\mathrm{Na}$ disciplina, exercícios de aplicação de conceitos teóricos permitem "ensaiar" produção de conhecimento: exigência é demonstrar compreensão adequada dos conceitos e tomar decisões equivalentes às que tomaria o pesquisador de determinada abordagem.

Por sua vez, o exercício de realização de uma pesquisa completa permite vivenciar a produção de conhecimento como processo de tomada de decisões. Ambas as situações vão na direção de se propiciar reflexão sobre a pesquisa e o papel do pesquisador. Na mesma linha, a discussão teórica, que ocorre paralelamente aos exercícios, permite abertura para a diversidade de possibilidades de pesquisa em psicologia.

A relação com a realidade brasileira e a reflexão sobre a intervenção em psicologia acontecem, a nosso ver, já na abordagem crítica do processo de pesquisa, com as características apontadas acima. Mas ficam reforçadas, na medida em que a discussão e, no caso do exercício prático de pesquisa, a escolha de objetos de pesquisa levam à reflexão sobre questões sociais relevantes. Nossa discussão no curso procura mostrar que relevância científica e relevância social de um problema são duas faces de uma mesma moeda, não há (ou não poderia ser considerada assim) uma relevância sem a outra.

Por fim, o princípio que aponta para a necessária participação do aluno fica respeitado quando objetivos e estratégias da disciplina exigem que o aluno se aproprie de conhecimentos existentes (teóricos e sobre as temáticas) e exercite a análise, a crítica e a proposição de investigações e/ou de intervenções. Isso é acompanhado por diversos momentos de decisão e escolha feitas pelos alunos. Eles decidem sobre tema de pesquisa e fazem todas as etapas, desde a colocação do problema.

Dessa forma, procuramos, ainda que no âmbito mais restrito de uma disciplina, pautar o desenvolvimento da ação pedagógica por princípios de formação. A reflexão sobre a articulação entre o fazer no cotidiano das atividades acadêmicas com os princípios apresentados nos parece essencial para superar visões tecnicistas de formação.

Assim, o projeto e a condução das disciplinas Modelos de Investigação I e II ministradas no curso de Psicologia da PUC/SP têm sido orientados por concepção de educação como nos ensinou Freire (1981): "problematizadora”, aquela que possibilita ao educando uma percepção transformadora

[...] capaz de perceber-se enquanto percebe a realidade que lhe parecia em si inexorável, é capaz de objetivála. [...] Desta forma, aprofundando a tomada de consciência da situação, os homens se "apropriam" dela como realidade histórica, por isto mesmo, capaz de ser transformada por eles (p. 85).

Entendemos o processo de formação, marcado pela interlocução e pela produção do saber, em que este sempre é uma prática produtiva e uma prática política. Assim, nossa atuação enseja que os estudantes e futuros psicólogos se qualifiquem para atuar em nossa sociedade de forma crítica e comprometida com sua transformação, considerando as necessidades sociais e os direitos humanos, o que vai ao encontro do que está expresso nos princípios de nossas Diretrizes Curriculares. 


\section{Referências}

Associação Brasileira de Ensino de Psicologia - ABEP. (2016). Linha do Tempo. Recuperado de http://www.abepsi.org. br/formacao/linha-do-tempo-da-psicologia/

BERNARDES, J. S. (2004) O debate atual sobre a formação em psicologia no Brasil: permanências, rupturas e cooptações nas políticas educacionais. (Tese de Doutorado). Programa de Estudos Pós-Graduados em Psicologia Social - PUCSP, São Paulo, Brasil.

Brasil. (2011). Resolução n.5 de 15 de março de 2011. Diretrizes Curriculares Nacionais da Psicologia. Brasília: Ministério da Educação, Câmara de Ensino Superior. Recuperado de http://portal.mec.gov.br/index.php?option=com docman\&view $=$ download\&alias $=7692-$ rces005-11pdf\&category_slug=marco-2011-pdf\&Itemid=30192

Conselho Regional de Psicologia de São Paulo. (1998). Jornal de Psicologia CRP-SP, 110. Recuperado de http://www. crpsp.org.br/portal/comunicacao/jornal_crp/110/ frames/fr_editorial.aspx

Freire, P. (1981) Pedagogia do Oprimido. (9a ed.). Rio de Janeiro: Paz e Terra. 